\title{
Raman spectroscopy methods for detecting and imaging supported lipid bilayers
}

\author{
Claire S. Sweetenham and Ioan Notingher* \\ Nanoscience Group, School of Physics and Astronomy, University of Nottingham, University Park, \\ Nottingham, UK
}

\begin{abstract}
We have developed a Raman microspectroscopy system optimised for studying supported lipid bilayers (SLB). This system combines the benefits of Raman spectroscopy with the high spatial resolution of confocal microscopy. Furthermore, the additional incorporation of an atomic force microscope (AFM) makes it possible to directly correlate chemical information with spatial features of samples at the nanoscale. We focus on the limits of this system for detecting a single SLB and imaging its microdomains, and employ surface-enhanced Raman spectroscopy (SERS) to improve the sensitivity achieved with Raman microspectroscopy.
\end{abstract}

Keywords: Supported lipid bilayers, atomic force microscopy, Raman microspectroscopy, surface-enhanced Raman spectroscopy

\section{Introduction}

Supported lipid bilayers (SLB) have found wide application as models of cellular membranes in biophysical research. SLB consist of amphiphilic molecules, or phospholipids, that can self-assemble into bilayer structures when deposited onto a hydrophilic support from an aqueous solution, either by vesicle fusion or the Langmuir-Blodgett technique [2]. Dipalmitoyl phosphatidylcholine (DPPC) and dioleoyl phosphatidylcholine (DOPC) are two types of phospholipid that are saturated and unsaturated respectively at room temperature due to their individual phase transition temperatures [5]. When combined in SLB these lipids form microdomains, phase-separated areas of ordered gel state and disordered liquidcrystalline state, which affect the structure and biological function of membranes and are an important feature in many cellular processes. These artificial membranes are well defined and stable under a variety of conditions, allowing characterisation with a broad range of physical methods.

Atomic force microscopy (AFM) is a non-destructive method used widely in science and technology to analyse all types of materials and surfaces. It is a scanning probe microscopy technique that employs the force between a probe and a sample to provide images of its surface with nanoscale resolution. The probe of an AFM is a tiny, sharp tip at the end of a cantilever that scans the surface of a sample. The forces that occur between the sample and the tip as it moves across the surface cause the cantilever to bend. This deflection is measured via the motion of a laser beam reflected from the back of the cantilever onto a position-sensitive photo-detector. These deflections are monitored by a feedback loop and used to produce a visual mapping of the surface, pixel by pixel.

\footnotetext{
* Corresponding author: Ioan Notingher, Nanoscience Group, School of Physics and Astronomy, University of Nottingham, University Park, Nottingham, NG7 2RD, UK. Tel.: +44 115951 5172; E-mail: ioan.notingher@ nottingham.ac.uk.
} 
AFM offers purely topographical and mechanical information. However its ability to detect deviations in the topography of a surface with angstrom precision makes AFM an invaluable tool for the characterisation of SLB and for studying the microdomains they form [4]. SLB are imaged with long triangular silicon nitride cantilevers with low spring constants, with the AFM in tapping mode and both the sample and probe immersed in water. A common feature of SLB is defects, or holes, which can be used to measure its thickness and therefore confirm its presence. In addition, the phase-separated microdomains that occur in mixed lipid SLB can be detected.

Raman spectroscopy is a well established, analytical tool that has been widely applied to biological and medical research. This optical technique relies on the inelastic scattering of monochromatic light from a material to generate a spectral fingerprint of a given sample. Raman spectra provide extensive information at the molecular level based on intrinsic molecular vibrations arising from specific components in the physio-chemical makeup of a material. It has many advantages for studying biological materials, for instance it allows label-free, real-time investigation with high chemical specificity.

Raman spectroscopy has the potential to offer detailed chemical and structural analysis of SLB. Previously, optical-trapping has enabled the detection of single phospholipid vesicles and signal-enhancing techniques can achieve the detection of multilayered or hybrid lipid structures [3,7]. However the sensitivity and spatial resolution that can be achieved with Raman spectroscopy can restrict its effectiveness at the micro- and nanoscale. A Raman microspectroscopy system, whilst possessing all the advantages of a conventional setup, has the ability to collect Raman scattering from a sample volume of less than $1 \mu \mathrm{m}$ in diameter. This technique is implemented by selective detection of Raman scattering through a pinhole by rejection of out-of-focus signal. Therefore any signal arising from any surrounding material, such as a substrate, that could obscure the weaker Raman signal from the sample can be reduced, allowing excellent depth discrimination.

\section{Materials and methods}

\subsection{Supported lipid bilayers}

Dipalmitoyl phosphatidylcholine (DPPC) and dioleoyl phosphatidylcholine (DOPC) are purchased as semi-synthetic lipids made from egg lecithin (Lipid Products, UK) and stored in chloroform and methanol solution. This $50 \mathrm{mg} / \mathrm{ml}$ lipid solution is placed in a small round-bottomed flask and dried under a stream of nitrogen until a thin lipid film is formed. The dried lipid film is rehydrated with water to give a milky solution which is then frozen in liquid nitrogen and thawed several times. Finally, the suspension is extruded through a polycarbonate filter with $100 \mathrm{~nm}$ diameter pores (Avanti Polar Lipids) to produce a monodisperse, unilamellar suspension of $100 \mathrm{~nm}$ diameter phospholipid vesicles.

The lipid vesicle solution is diluted in water and deposited on a polished sapphire substrate (PI-KEM, UK) that has been annealed at high-temperature and treated with magnesium chloride. The sample left for $3 \mathrm{~h}$, in which time the lipid vesicles fuse across the substrate to form a bilayer. The sample is washed with water to remove any excess lipids and left in a hydrated state, resulting in a unilamellar supported phospholipid bilayer formed between two layers of water.

\subsection{Atomic force microscopy}

Samples are imaged with an atomic force microscope (AFM) (Nanowizard, JPK Instruments) with piezoelectric $x y$-stage and tip-assisted optics (TAO) module fitted to an inverted microscope (IX71, 
Olympus). The AFM is used in tapping mode in water, with triangular non-conductive silicon nitride probes (Veeco) with a resonant frequency of $12-24 \mathrm{kHz}$ and a spring constant of $0.06 \mathrm{~N} / \mathrm{m}$. Images are recorded on software accompanying the AFM and processed and analysed with WSxM software (Nanotech Electronica).

\subsection{Raman microspectroscopy}

Raman measurements are performed on a home-built Raman microspectroscopy system optimised for characterising and studying SLB (Fig. 1a). This system combines a Raman spectrometer, microscope and confocal system to provide high spatial resolution and signal collection from small sample volumes. The

(a)

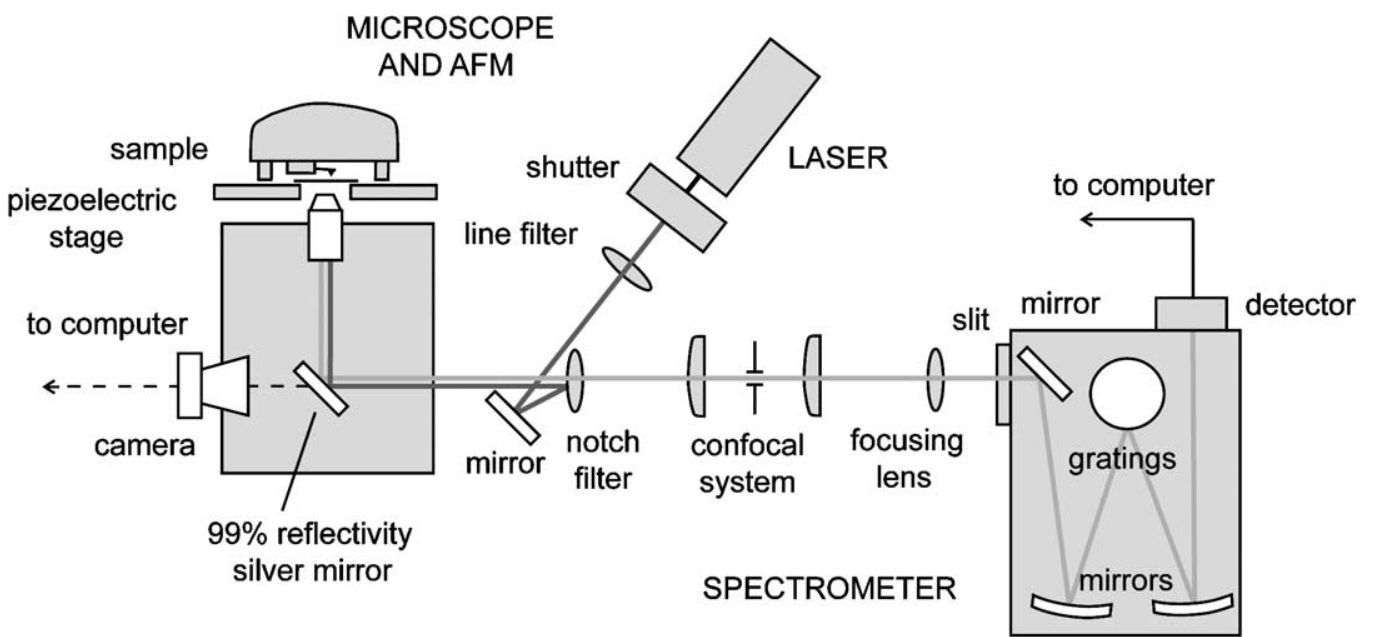

(b)

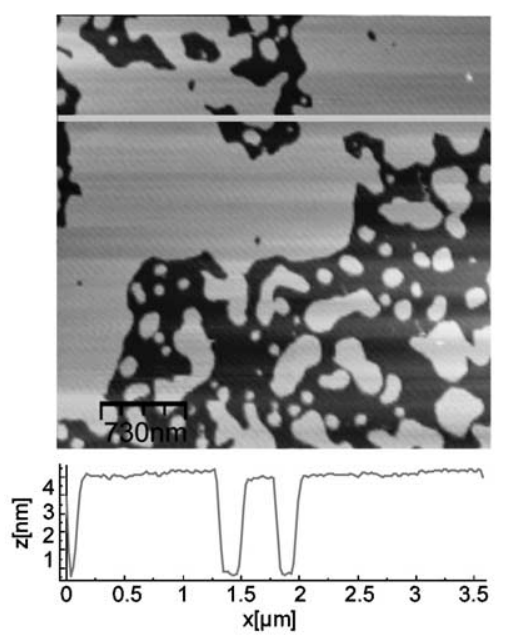

(c)
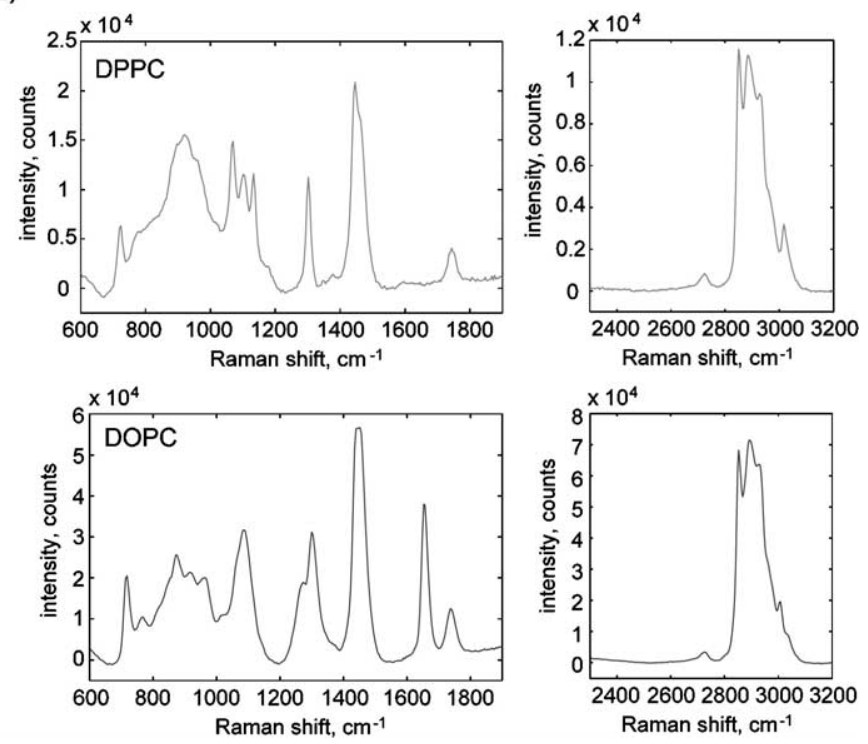

Fig. 1. (a) Schematic of combined Raman microspectroscopy and AFM system; (b) AFM image and line profile of a DPPC SLB; (c) Raman spectra of DPPC and DOPC in multilayered structures. 
system was designed to collect the maximum amount of Raman scattering possible whilst minimising any background and noise to optimise optical throughput through the system.

An inverted microscope (IX71, Olympus) is employed to avoid disturbances to samples immersed in liquid and also to allow simultaneous imaging with an atomic force microscope (AFM), which is not possible with an upright microscope. A $20 \mathrm{~mW} 532 \mathrm{~nm}$ continuous wave diode-pumped green laser (Laser 2000, UK) is directed through the microscope by a dichroic mirror (Semrock) and focused onto the sample by a water-immersion 1.2NA $60 \times$ objective (Olympus). Raman scattering is collected by the same objective and directed through a confocal system containing a $75 \mu \mathrm{m}$ pinhole (Thorlabs, UK) to enhance spatial resolution, which is about $3 \mu \mathrm{m}$ in the optical axis and less than $1 \mu \mathrm{m}$ in the sample plane. The resultant signal is focused into a $\mathrm{f} / 4$, double Czerny-Turner Raman spectrometer equipped with a $600 \mathrm{l} / \mathrm{mm}$ ruled grating and a back-illuminated CCD (Andor Technologies, UK). Measurements are recorded with accompanying software and processed and analysed with Matlab (Mathworks, UK).

\section{Results}

Single supported lipid bilayers (SLB) have been formed on sapphire and imaged with atomic force microscopy (AFM) at room temperature. The presence of single lipid SLB of dipalmitoyl phosphatidylcholine (DPPC) and dioleoyl phosphatidylcholine (DOPC) has been confirmed via holes in the structure, topography line profiles indicating a bilayer height of $4.1 \mathrm{~nm}$ (Fig. 1b). DPPC and DOPC have also been combined in SLB, again confirmed with a bilayer height of $4.1 \mathrm{~nm}$. These SLB contain microdomains of gel state DPPC protruding $0.9 \mathrm{~nm}$ from liquid-crystalline DOPC, due to phase transition temperatures of $41^{\circ} \mathrm{C}$ and $-20^{\circ} \mathrm{C}$, respectively [5].

Following confirmation of the presence and characterisation of SLB, the inclusion of an AFM in the Raman microspectroscopy system allows the collection of Raman scattering from the SLB at exactly the same position on the sample. Raman spectra of DPPC and DOPC in multilayered structures have been measured with the Raman microspectroscopy system (Fig. 1c) with laser powers and exposure times that have been shown to have no significant effect on the physical or chemical structure of the lipids. Notable Raman bands include carbon-carbon double bond in-plane bending at $953 \mathrm{~cm}^{-1}$ and stretching at $1641 \mathrm{~cm}^{-1}$, only present in the spectra of liquid crystalline DOPC at room temperature.

The Raman microspectroscopy system does not have the sensitivity to detect a single SLB. The spatial resolution of the setup has been optimised by a water-immersion, high numerical aperture objective and confocal system to improve the collection of scattering from small samples. Also, sapphire has been used as a substrate for the SLB to minimise background scattering, as it has very little Raman scattering and bands itself. However the scattering from SLB is too weak to be detected with the current system.

\section{Conclusions and future work}

A Raman microspectroscopy system containing an inverted microscope and confocal system with good spatial resolution has been developed and characterised for supported lipid bilayers (SLB). The system also includes an atomic force microscope (AFM) to allow simultaneous Raman spectroscopy and AFM measurements. Dipalmitoyl phosphatidylcholine (DPPC) and dioleoyl phosphatidylcholine (DOPC) SLB have been created and imaged with AFM. Single and mixed lipid SLB have been characterised by topography line profiles, measuring a bilayer height of $4.1 \mathrm{~nm}$. SLB containing both DPPC and DOPC formed microdomains that protrude $0.9 \mathrm{~nm}$ from the rest of the bilayer. Raman spectra of 
DPPC and DOPC in multilayered structures have been measured and significant Raman bands have been identified. However the detection of a single SLB is not possible with this system and the sensitivity currently achievable.

Signal enhancement techniques have been considered to enhance the scattering from SLB. Surfaceenhanced Raman spectroscopy (SERS) exploits the enhancement of scattering close to rough metallic surfaces, arising from locally enhanced electromagnetic fields caused by surface plasmon resonances, and has been identified as an appropriate addition to the current setup. However for it to be suitable for both the Raman microspectroscopy system and SLB, the SERS substrate must be transparent and have the right conditions for SLB formation and imaging. We are currently developing SERS-active substrates formed from silver thin films evaporated onto a deposition mask of close-packed polystyrene nanospheres [1] on sapphire. There is uncertainty concerning the level of signal enhancement this technique can provide and whether the localised heating associated with SERS may damage SLB [6]. Therefore the SERS enhancement gained from different lipids will be investigated, in single and mixed lipid SLB, and the possibility of causing heat damage to SLB. In addition, correlation between SERS and imaging has to be established.

\section{Acknowledgement}

We thank the UK Engineering and Physical Sciences Research Council (EPSRC) for financial support.

\section{References}

[1] L. Baia, M. Baia, J. Popp and S. Astilean, Gold films deposited over regular arrays of polystyrene nanospheres as highly effective SERS substrates from visible to NIR, Journal of Physical Chemistry B 110 (2006), 23982-23986.

[2] E.T. Castellana and P.S. Cremer, Solid supported lipid bilayers: From biophysical studies to sensor design, Surface Science Reports 61 (2006), 429-444.

[3] D.P. Cherney, J.C. Conboy and J.M. Harris, Optical-trapping Raman microscopy detection of single unilamellar lipid vesicles, Analytical Chemistry 75 (2003), 6621-6628.

[4] Y.F. Dufrêne and G.U. Lee, Advances in the characterization of supported lipid films with the atomic force microscope, Biochimica et Biophysica Acta 1509 (2000), 14-41.

[5] R. Koynova and M. Caffrey, Phases and phase transitions of the phosphatidylcholines, Biochimica et Biophysica Acta 1376 (1998), 91-145.

[6] E.C. Le Ru and P.G. Etchegoin, Vibrational pumping and heating under SERS conditions: fact or myth?, Faraday Discussions 132 (2006), 63-75.

[7] C. Lee and C.D. Bain, Raman spectra of planar supported lipid bilayers, Biochimica et Biophysica Acta 1711 (2005), 59-71. 


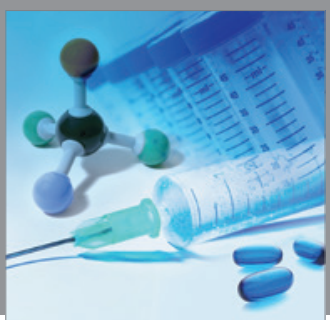

International Journal of

Medicinal Chemistry

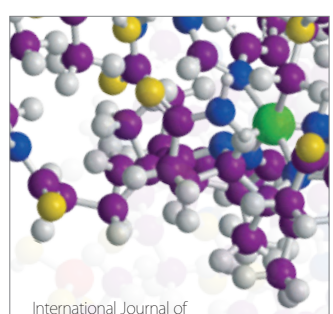

Carbohydrate Chemistry

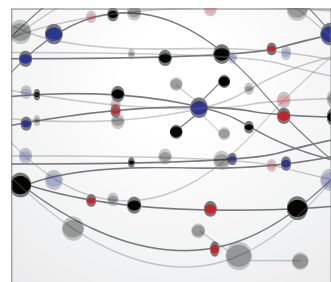

The Scientific World Journal
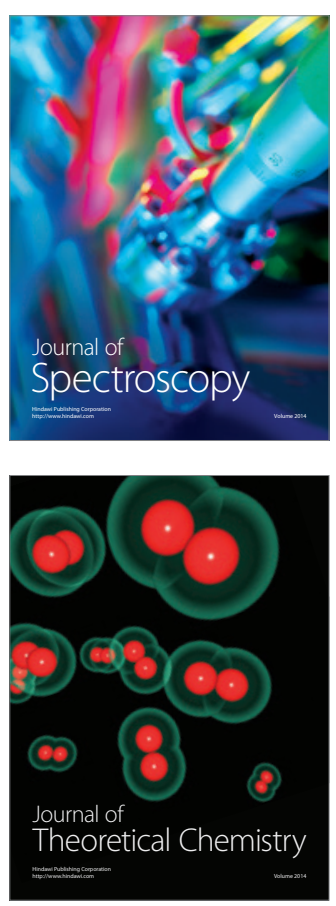
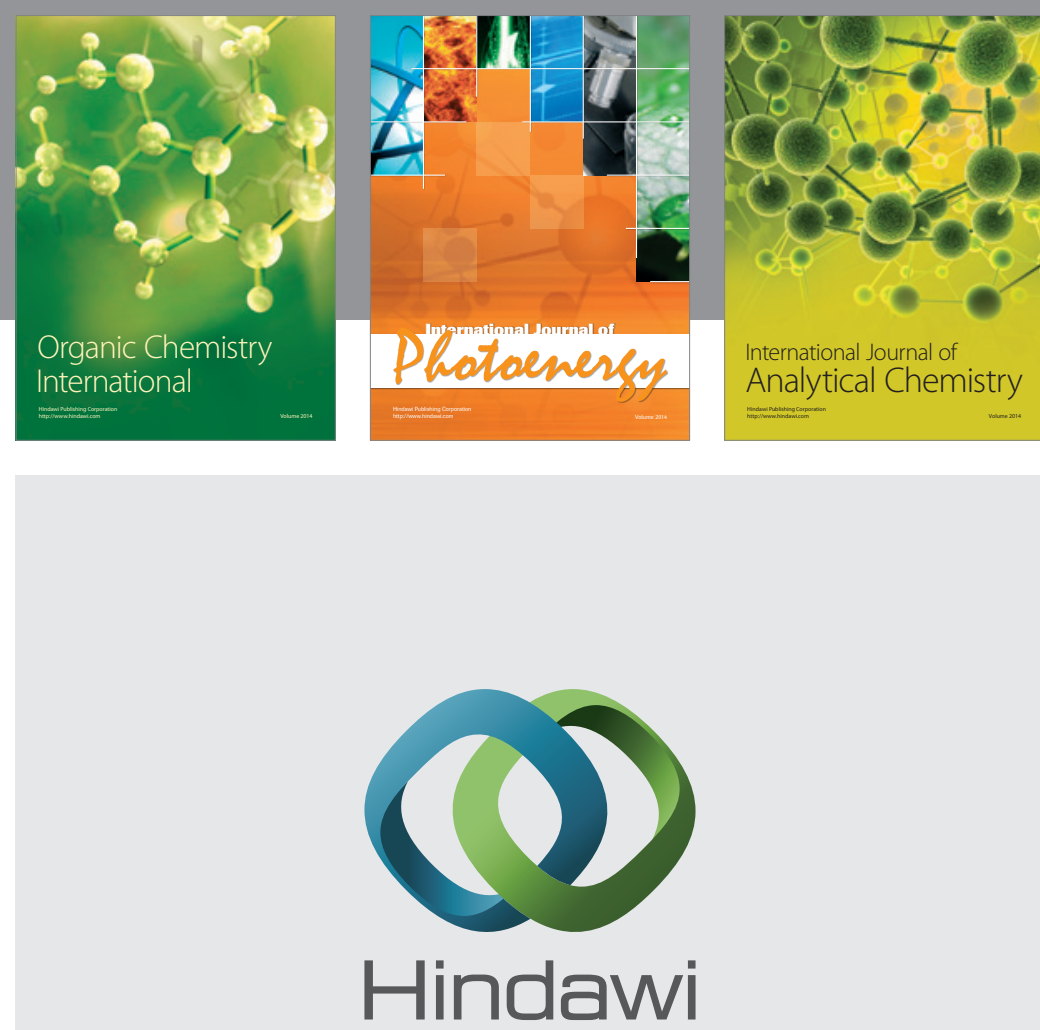

Submit your manuscripts at

http://www.hindawi.com
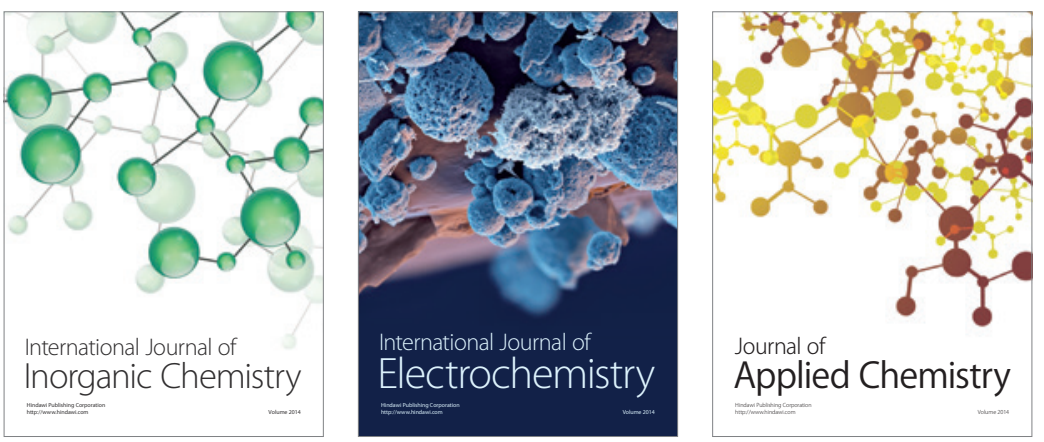

Journal of

Applied Chemistry
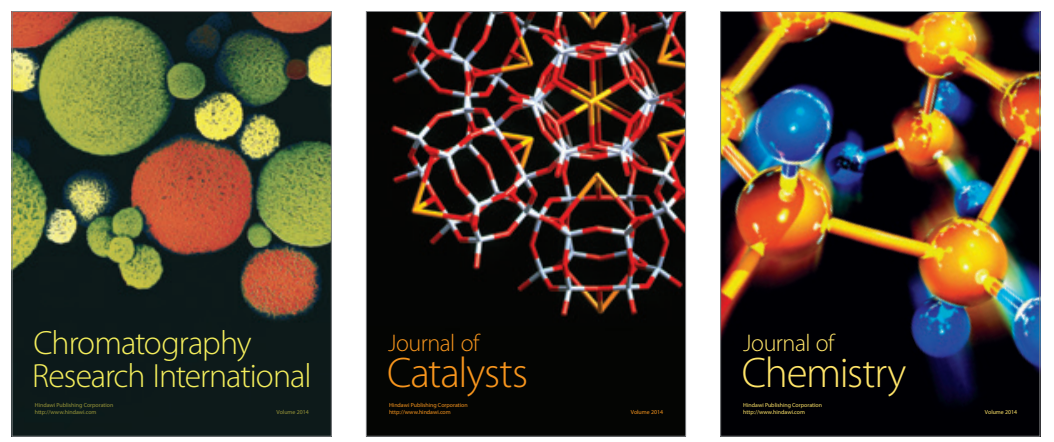
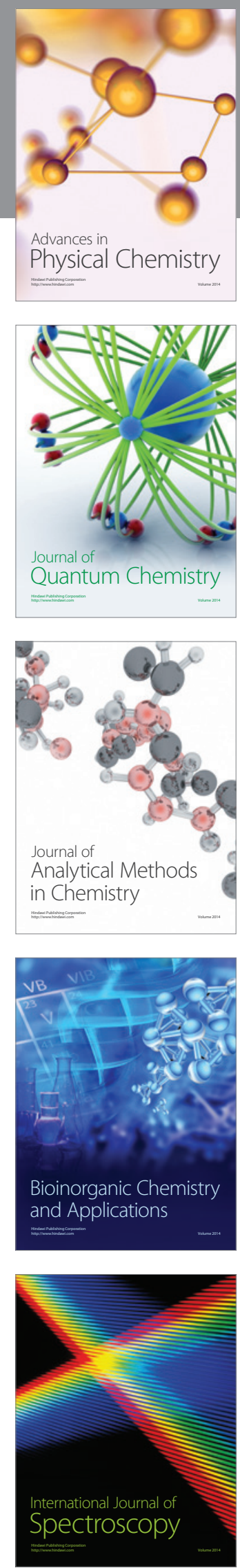